\title{
Límites en la capacidad de procesamiento y déficit atencional en niños hiperactivos
}

\author{
AGUSTÍN ROMERO, CONCHA LÓPEZ y ASCENSIÓN GARRIGA
}

Universidad de Murcia

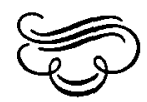

Resumen

El déficit atencional en niños hiperactivos ba sido investigado a partir de las teorias tradicionales de la atención. Este trabajo incorpora las recientes teorias de limitaciones en la capacidad de procesamiento mediante un paradigma de doble tarea (visual y auditiva). Se manipula el nivel de dificultad de la tarea secundaria y se comprueba su efecto en niños biperactivos $(n=28)$ y normales $(n=28)$ de $2 .^{\circ}-3 .^{\circ}$ y $4 .^{\circ}-6 .^{\circ}$ cursos de EGB. Los resultados permiten sostener que al aumentar la dificultad de la tarea secundaria, los hiperactivos de $4 .^{\circ}-6 .^{\circ}$ tienen peor ejecución que los controles de los mismos cursos y edad, mientras que en $2 .^{\circ} \cdot 3 .^{\circ}$ ocurre lo contrario. Los biperactivos muestran una mayor inestabilidad y labilidad en la asignación de recursos y/o en los limites de capacidad de procesamiento.

Palabras clave: Capacidad de procesamiento, Atenciön, Hiperactividad, Dificultades de aprendizaje.

\section{Processing capacity limits and attentional deficit in hyperactive children}

\section{Abstract}

The attentional deficit in byperactive children has been researched from attentional traditional theories. The processing limitaions capacity theories are incorporated in this paper by means of a dual task (visual and auditory) paradigm. The level of difficulty of the secondaty task is manipulated and its effect in byperactive $(n=28)$ and controls $(n=28), 2 .^{\circ}-3 .^{\circ}$ and $4 .^{\circ}-69^{\circ}$ EGB graders is verified. The results allow us to say that when the difficulty of the secondary task is increased the byperactives $\left(4 .^{\circ} \cdot 6^{\circ}\right.$ graders) bave then poorer performance than controls of the same courses and ages. Whereas in $2 .^{\circ}-30^{\circ}$ graders the opposite occurs. The byperactives show a greater instability and weakness in the resource allocation and/or in the processing capacity limitations.

Key words: Processing capacity, Attention, Hyperactivity, Leaming disabilities.

Direcciōn de los autores: Dr. Agustín Romero Medina, Departamento de Metodología y Análisis del Comportamiento. Facultad de Filosofia, Psicología y CC.EE., Universidad de Murcia, 30071 Murcia. 


\section{INTRODUCCION}

Uno de los problemas clínicos más habituales en las aulas es la presencia de niños cuyo comportamiento suele calificarse como «excesivo» en cuanto a actividad e impulsividad, y como emínimo en lo que respecta a un escaso control en la inhibición adecuada de conductas y escasa concentración en tareas escolares. Se trata del síndrome de hiperactividad, el cual, según las últimas descripciones nosológicas (vid. DSM III, 1980) incluye un importante déficit atencional, cuyo estudio e investigación va adquiriendo últimamente considerable interés en la literatura sobre el tema, tanto en revisiones teóricas (Douglas y Peters, 1979; Prior y Griffin, 1985; Rosenthal y Allen, 1978) como en estudios experimentales (Ackerman, Anhalt, Dykman y Holcomb, 1986; Hiscock, Kinsbourne, Caplan y Swanson, 1979; Prior, Sanson, Freethy y Geffen, 1985; Sergeant y Scholten, 1985a, b).

Dada esta creciente importancia concedida al déficit atencional como agente etiológico relevante en las dificultades de aprendizaje en general y en la hiperactividad en particular, conviene revisar más en profundidad las investigaciones al respecto, sobre todo en lo relativo a investigaciones empíricas y experimentales basadas en las aportaciones recientes en métodos y teorías de la investigación básica en procesos atencionales.

Así como en otros aspectos conductuales, psicométricos o neuropsicológicos esos procesos parecen cada vez mejor delimitados (Vid. López, 1986, para revisión), en el síndrome de hiperactividad, sin embargo, y en lo que respecta a la conceptualización y características del déficit atencional, la literatura empírica se encuentra plagada de notables contradicciones, confusiones y problemas teóricos y metododlógicos, todo lo cual hace difícil llegar a tener una idea clara sobre la función de este déficit en la etiología, diagnóstico y tratamiento de la hiperactividad.

Prior et al. (1985) han resumido los principales problemas existentes en torno a la investigación experimental sobre el déficit atencional en varios puntos que nosotros agrupamos en dos: los de tipo teórico y los metodológicos.

Desde el punto de vista teórico o conceptual se señala, en primer lugar, y siguiendo con ello a Douglas y Peters (1979) y Taylor (1980), una conceptualización imprecisa del déficit atencional, con énfasis diferente, según autores, en aspectos tales como atención selectiva, atención sostenida, activación focalizada, control de impulsos, distractibilidad, etc. Esta cuestión sería a su vez consecuencia, a nuestro juicio, de una gran inestabilidad en las teorías y modelos atencionales surgidos de la investigación en este campo. Inestabilidad debida, muy probablemente, al rápido cambio y desarrollo que en los últimos años se está produciendo en el estudio de la atención (De Vega, 1984; Eysenck, 1982; Parasuraman y Davies, 1984; Ruiz Vargas y Botella, 1981) por la influencia del auge cognitivo en psicología y que genera una situación por la que los principios explicativos y los modelos atencionales utilizados para el análisis del déficit atencional quedan desfasados antes de su aplicación o investigación clínica.

En cuanto a los problemas metodológicos, se citan algunos, tales como: muestras mal definidas y seleccionadas (Rosenthal y Allen, 1978), ausencia en algunos casos de grupos de control, influencia no especificada de los parámetros evolutivos normales (Prior et al., 1985), escasa delimitación de tareas y de condiciones de tareas experimentales, etc. i. Los primeros trabajos de tipo experimental sobre los aspectos cognitivos del 
déficit atencional en hiperactivos proceden de los grupos de Douglas, en Canadá, y Prior, en Australia, durante los años 70 . Desde entonces, los resultados obtenidos en la no muy abundante investigación experimental se podrían resu. mir, según Douglas y Peters (1979) en dos conclusiones: (1) mayor dificultad de los niños hiperactivos en sostener la atención en tareas que requieren demandas especiales de esfuerzo concentrado, autosostenido y autodirigido (por ejemplo, en tareas experimentales de vigilancia, de cierta duración) y (2) mayor dificultad en inhibir la responsividad impulsiva sobre tareas o en situaciones sociales que requieren esfuerzo focalizado, reflexivo y organizado. O sea, déficit en atención sostenida y en atención focalizada por pobre control inhibitorio. Sin embargo, no se han hallado diferencias con normales en atención selectiva auditiva (Hiscock et al., 1979; Prior et al., 1985) y visual (Sergeant y Scholten, 1985a,b), y tampoco los estudios sobre distractibilidad, en contra de lo que intuitivamente pudiera pensarse, han sido muy concluyentes (Prior et al., 1985; Peters, 1977; Rosenthal y Allen, 1978).

Así, pues, el déficit en atención sostenida, el pobre control inhibitorio junto con una activación o «arousalı pobremente modulada, forman los tres factores atencionales fundamentales en el modelo de Douglas y Peters (1979) sobre el déficit atencional en hiperactivos. Estos tres elementos iniciales, junto con sucesivas experiencias de fracasos y conductas de evitación llevan a un aumento, continuo con la edad, de problemas de concentración, impulsividad y motivación en la resolución de problemas, así como en otras tareas escolares complejas.

Sin embargo, un aspecto cognitivo de la atención prácticamente inexplorado .. en el déficit atencional hiperactivo ha sido el de la limitación de capacidad de procesamiento. A partir de la década de los 70 empezó a tomar consistencia un nuevo enfoque de la atención por el cual ésta se viene a considerar no ya en su dimensión de mecanismo de selección de información - primeras teorías - de filtro a partir de Broadbent, Treisman, Deutsch y Deutsch, etc.- o en su dimensión más psicofisiológica de activación, alerta o reflejo de orientación, sino más bien como mecanismo que limita la capacidad de procesamiento.

Los trabajos experimentales sobre el déficit atencional antes mencionados toman como marcos teóricos de referencia los siguientes: (1) las teorías atencionales derivadas de los primeros modelos etápicos del procesamiento de información y se interesan por cuestiones tales como las limitaciones estructurales en el procesamiento y, por tanto, en el alocus» de la selección de información, sean en las primeras etapas de procesamiento - modelos de aselección tempranas, de Broadbent (1958) - en las últimas - modelos de aselección tardíau, de Welford $(1952,1968)$, Deutsch y Deutsch (1963), Norman $(1968,1976)$, Duncan (1980) - o bien en múltiples niveles - modelos de sselección múltiple», de Broadbent (1970), Johnston y Heinz (1978) y Treisman $(1960,1964)$, y (2) las teorías atencionales de origen psicofisiológico relacionadas con los aspectos de intensividad y activación, es decir, con la focalización prolongada o sostenida del sistema de procesamiento sobre determinados estímulos o tareas. La intensividad atencional ya ha sido relativamente bien documentada como componente del déficit atencional. La selectividad atencional, según la revisión antetáor, apenas resulta alterada en los hiperactivos y además, a nivel teórico ha ido perdiendo fuerza ante la gran dificultad por llegar a un acuerdo sobre las caractarísticas generales de la misma, por lo cual surgieron los modelos de limitación de capacidad.

Los modelos atencionales de limitación de capacidad tienen su primer mo- 
mento de expansión a partir del trabajo seminal de Kahneman (1973). Según Allport (1980), estos modelos parten del supuesto principal según el cual el procesamiento humano de información tiene una capacidad limitada en sus funciones. Dicha limitación no es estructural o etápica, sino global e inespecífica y la capacidad de procesamiento o esfuerzo», «energía» o «atención» es de propósito general y susceptible de ser asignada a todos y cada uno de los procesos mentales. Así, cuando un sujeto realiza más de una tarea simultáneamente, la capacidad o «recursos» de procesamiento se asignarán a dichas tareas de tal modo que si las demandas de las tareas exceden los límites de capacidad disponible, la ejecución será peor.

Las teorías atencionales de capacidad podrían agruparse en dos grandes tendencias: los modelos de recursos y asignación de capacidad y los modelos de capacidad variable. Dentro de los primeros hay que citar el modelo de capacidad como procesador central de propósito general de Kahneman (1973), el modelo de capacidad inespecífica limitada por datos y por recursos, de Norman y Brobow (1975) y el modelo de recursos múltiples de Navon y Gopher (1979, 1980). En general, se trata de modelos que se interesan por la asignación o distribución de recursos de procesamiento y por los límites funcionales de dicha asignación según las demandas de las tareas que simultáneamente realiza el sujeto. Por otra parte, los modelos de capacidad variable parten de que los procesos cognitivos varían en gasto de capacidad y recursos a lo largo de dos extremos: escaso o nulo y gran gasto de recursos o capacidad. Una tarea puede requerir distintos niveles de capacidad de procesamiento según el grado de experiencia, práctica o aprendizaje previo sobre la misma. De este tipo, los modelos más conocidos son los de procesamiento automático versus controlado de Shiffrin y Schneider (1977), automático versus consciente de Posner y Snyder (1975) y automático versus «con esfuerzo» (effortful), de Hasher y Zacks (1979).

Vamos a centrar la aportación empírica de este trabajo en alguno de los aspectos de los modelos de recursos y asignación de capacidad de procesamiento. Casi todos ellos han sido puestos a prueba mediante paradigmas experimentales de doble tarea, es decir, situaciones en las que, variando el nivel de dificultad de una de las tareas o dando instrucciones de focalización en una de ellas, se puede analizar la asignación de recursos y los límites de capacidad de procesamiento. Aunque no son pocas las insuficiencias y problemas criticados en estos modelos (vid. Allport, 1980; Eysenck, 1982), podemos suponer que si en un paradigma de doble tarea incrementamos el nivel de dificultad de la tarea secundaria, de acuerdo con Kahneman (1973), ello implica que dicha tarea exige mayor demanda de recursos. Si el sistema está ocupado además con la realización simultánea de otra tarea, es posible que al aumentar la demanda de recursos (dificultando la tarea secundaria) se llegue al límite de capacidad y, por tanto, la ejecución en la tarea principal se vea interferida por aquélla (con ello nơ es que aceptemos sin más el Principio de Complementariedad de Norman y Brobrow (1975), el cual implica una capacidad de límite fijo, sino que sólo lo admitimos como hipótesis, y, por tanto, como una posibilidad entre otras). La interferencia o disminución de la ejecuación en la tarea principal por aumento de dificultad de la tarea secundaria sería señal de que, al exceder los límites de capacidad, el sistema sustrae recursos asignados a la tarea principal.

Si siguiéramos este razonamiento al aplicar un paradigma de doble tarea similar a niños hiperactivos, cabe la hipótesis de que en éstos aparezca una ejecución diferenre a la de niños normales: en los hiperactivos se observaría una ma- 


\section{1}

yor interferencia por menor capacidad o por mayor limitación de la misma.

De este modo, y como tercer supuesto, se podría pensar a la luz del modelo de Douglas y Peters (1979), que las experiencias continuas de fracaso y conductas de evitación por los tres déficits atencionales mencionados, según pasan los años durante la infancia ocasionarán un desarrollo anómalo en el conocido (Hashet y Zacks, 1979; Prior et al.. 1985) aumento de capacidad de procesamiento con la edad. Es decir, comparando a niños de dos grupos de edad, por ejemplo 7-8 años y 9-11 años, el aumento en capacidad de procesamiento con la edad será menor en hiperactivos que en normales.

A partir de estos supuestos intentaremos comprobar: (1) que el aumento de dificultad de la tarea secundaria hace empeorar la ejecución de la tarea principal en un paradigma de doble tarea y, por consiguiente, que este parámetro puede utilizarse como indicador de los límites en la capacidad y recursos inespecíficos de procesamiento; (2) que al comparar a hiperactivos con normales en el paradigma citado, los primeros exhibirán una peor ejecución de interferencia en la tarea principal que los segundos, demostrando así la existencia de un déficit atencional basado en una menor capacidad y recursos inespecíficos de procesamiento, y (3) que al comparar dos grupos de edad en hiperactivos y en normales, estos últimos mostrarán con la edad una mejor ejecución como consecuencia de un desarrollo normal en el aumento de capacidad y posibilidades de asignación de recursos, mientras que los hiperactivos no poseerán tanto desarrollo en dichas características atencionales.

Para la verificación de tales hipótesis utilizaremos un paradigma de doble tarea ${ }^{1}$ en el cual la tarea principal se basa en el paradigma de búsqueda visual de letras o rastreo (v. gr. Bouma, 1978), y en la cual el sujeto tiene que tachar una letra objetiva en un conjunto de filas de letras diferentes (tarea que también sería similar, a nivel aplicado, al Test de Tachado de Zazzo, 1971). La tarea secundaria concurrente consiste en la presentación auditiva de grupos de dígitos con objetivo distinto en cada ensayo (grupos de dos o tres dígitos en cada ensayo, añadiendo en cada presentación un dígito nuevo, quitando uno de los presentados en el ensayo anterior y pidiendo al sujeto que evoque verbalmente el dígito nuevo).

Ambas tareas exigen recursos estructurales diferentes (visuales y auditivos) y por ello hemos de suponer que la posible interferencia será fundamentalmente de capacidad. En la segunda tarea se manipula el nivel de dificultad asignando a los sujetos a dos grupos según se les presente ensayos de dos o tres dígitos, respectivamente. Al aumentar el nivel de dificultad suponemos que se recarga la capacidad de la memoria de trabajo (ya de por sí ocupada en la tarea principal). Por último, también partimos de que ambas tareas entrarían en lo que Shiffrin y Schneider (1977) denominan «procesos controlados», es decir, con gasto de capacidad y sin que la práctica en la tarea (que es escasa por su corta duración) permita su transformación en procesos automáticos.

\section{METODO}

\section{Sujetos}

Intervinieron un total de 56 niños y niñas (aproximadamente un $85 \%$ de niños, dadó que la epidemiología del síndrome hiperactivo señala una mayor 
incidencia en niños que en niñas), de los cuales 28 pertenecen al grupo de hiperactivos y otros 28 forman el grupo control de niños normales no hiperactivos de similar edad y curso. A su vez, cada uno de estos subgrupos está formado por niños de dos niveles de edad y curso: los más jóvenes son de $2 .^{\circ}$ y $3 .^{\circ}$ de EGB (7.9 años: $\bar{X}=8.3$, desv. tip. $=0.65)$ y los mayores de $4 .^{\circ}$ y $6 .^{\circ}$ de EGB $(10-11$ años; $\overline{\mathrm{X}}=10.8$, desv. tip. $=1.47)$. Además, la mitad de cada uno de estos subgrupos de edad era asignado a cada una de las dos tareas secundarias (de dos y tres dígitos, respectivamente), resultando de esta distribución según hiperactividad, curso escolar y nivel de dificultad, un total de ocho grupos de siete niños cada uno.

La selección de los niños hiperactivos se realizó a partir de los siguentes criteterios clínicos y psicométricos:

Como criterios clínicos, se descartaron aquellos niños en cuya historia aparecieran, como alteraciones primarias, las de retraso mental, psicosis infantil, transtornos de conducta y alteraciones neurológicas específicas. Con padres y profesores se efectuaron entrevistas para determinar si los niños preseleccionados pa. ra el grupo experimental mostraban un comportamiento impulsivo, distraído, inatento e inquietante hasta el punto que cumpliera los siguientes criterios: (1) maniféstar todos y cada uno de los síntomas concretados en el Cuestionario de Hiperactividad (Vid. apéndice 1), confeccionado por nosotros y siguiendo los cifterios del DSM III al respecto; (2) persistencia de dichos síntomas como mínimo durante un año y aparición de los mismos antes de los 7 años de edad, y (3) coincidencia de padres y maestros en los mismos síntomas. Comparados con los del DSM III, estos criterios han sido deliberadamente más restrictivos con el fin de obtener una muestra más homogénea.

Como pruebas psicométricas se han utilizado varias ${ }^{2}$, pero solamente la Escala WISC de inteligencia sirvió como criterio de selección para dẹscartar casos posibles de retraso mental.

\section{Aparatos y material estimular}

Para la tarea principal se proporcionaba al sujeto un folio (hoja de respuesta de la tares de tachado), en la cual figuraba una matriz de $25 \times 40$ letras mayúsculas, o sea, 1.000 letras formadas por la repetición aleatoria de las letras A, B, C, D, E, F, G, H, siendo «A» la letra objetivo que el sujeto debía tachar con una raya hecha con lápiz. Previamente a la matriz de prueba se presentaba otra más pequeña de $25 \times 3$ letras como práctica.

La tarea auditiva y las instrucciones para su realización se grabaron en un cassette stereo, el cual sirvió también para su aplicación a los sujetos. En esta tarea se presentaban dos ensayos de práctica y diez de prueba. Cada ensayo consistía en la exposición de dos o tres dígitos (según fuese la tarea secundaria efácil» 0 «difícil») con intervalos de un segundo entre dígitos y con intervalo entre ensayos de duración aleatoria, pero de tal forma que el tiempo desde el primero al décimo ensayo de prueba fuese de dos minutos (que era justo el tiempo en que simultáneamente el sujeto debía estar realizando la tarea de tachado). Los estímulos empleados fueron, en la tarea de dos dígitos: 4-2, 2-5, 5-1, 5-3, 3-6, 2-6, 5-2, 2-1, 1-3, 1-5; y en la de tres dígitos: 4-3-2, 4-2-5, 2-5-1, 1-5-3, 3-1-6, $6-3-2,2-6-5,1-6-2,2-3-1$ y $1-5-2$. 
Procedimiento

Las tareas fueron realizadas en una sala del colegio ${ }^{3}$, estando el experimentador junto al niño. Para evitar diferencias en la aplicación de consignas e instrucciones, éstas fueron grabadas previamente y reproducidas mediante cassette. Tras el período de entrenamiento o prácticas con ambas tareas y comprobada la comprensión de las mismas por el sujeto, se iniciaba la fase de prueba accionando el cassette a partir del primer ensayo de dígitos a la vez que el niño realizaba desde ese momento la tarea de tachado. A partir de la presentación del segundo ensayo de dígitos, el niño debía decir, inmediatamente después de haberlos oído y en voz alta, qué dígito nuevo había aparecido con respecto a la anterior presentación, y así en los sucesivos ensayos. El máximo de aciertos en la tarea auditiva era, pues, de nueve. En cuanto a la prueba de tachado, ésta se interrumpía al finalizar la presentación auditiva del décimo ensayo y la puntuación obtenida era el resultado de aplicar la fórmula $P=(A / 125) \times 10$, donde A es el número de letras localizadas correctamente y 125 es el número de veces que aparece en la matriz la letra objetivo «A».

En el estudio piloto se intentó aumentar la dificultad de la tarea secundaria de dos formas: pidiendo al sujeto no una evocación verbal de cada dígito nuevo, sino su anotación escrita en la misma hoja de la tarea de tachado, y también utilizando cuatro dígitos en cada ensayo. En ambośs casos la ejecución era prácticamente nula, por lo cual decidimos la aplicación de la tarea secundaria en sus dos niveles de dificultad tal y como ha sido descrita.

\section{Diseño experimental y análisis estadístico}

Se han manejado tres variables intersujeto: hiperactividad (grupo de hiperactivos, grupo central), curso $\left(2 .^{\circ}-3 .^{\circ}, 4 .^{\circ}-6^{\circ}\right)$ y dificultad de tarea secundaria («fácil» con dos dígitos y «difícil» con tres dígitos). Se trata, pues, de un diseño factorial intersujeto $2 \times 2 \times 2$, de forma que a cada uno de los ocho grupos resultantes se han asignado siete sujetos que reunían tales características. Puesto que disponemos de tres puntuaciones por cada sujeto, es decir, aciertos ponderados en la tarea principal, aciertos en la tarea secundaria y la suma de ambos, se han realizado con cada una de ellas el correspondiente Análisis de Varianza factorial intersujeto $2 \times 2 \times 2$ (mediante el paquete estadístico informatizado BMDP4V del Centro de Proceso de Datos de la Universidad de Murcia) cuyos resultados exponemos a continuación.

\section{RESULTADOS}

En la Tabla 1 podemos observar la distribución media (y dispersión) de los sujetos según las tres puntuaciones analizadas y en cada uno de los ocho grupos.

Podemos apreciar en la Figura 1 cómo el grupo central se ajusta a las predicciones propuestas en las tres puntuaciones, o sea, estos niños obtienen mejor puntuación en la tarea de dos dígitos que en la de tres, tanto los de curso inferiores como los superiores y además los de $20^{\circ}-3 .^{\circ}$ consiguen peor rendimiento que los de $4^{\circ}-6 .^{\circ}$ 


\section{4}

TABLA I

Distribución por grupos de la ejecución según puntuaciones medias ( $y$ dispersión) en la tarea principal $\left(P_{1}\right)$. tarea secundaria $\left(P_{2}\right)$ y en la suma de ambas $\left(P_{t}\right)$.

\begin{tabular}{|c|c|c|c|c|c|c|c|}
\hline \multirow{2}{*}{ Curso } & \multirow{2}{*}{$\begin{array}{l}\text { Dificultad } \\
\text { tarea secundaria }\end{array}$} & \multicolumn{3}{|c|}{ Grupo Control } & \multicolumn{3}{|c|}{ Grupo Hiperactivos } \\
\hline & & $P_{1}$ & $P_{2}$ & $\mathrm{P}_{\mathrm{r}}$ & $P_{1}$ & $P_{2}$ & $P_{t}$ \\
\hline \multirow[b]{2}{*}{$2 .^{\circ} \cdot 3 .^{\circ}$} & 2 dígitos & $\begin{array}{c}2.50 \\
(0.83)\end{array}$ & $\begin{array}{c}6.28 \\
(1.79)\end{array}$ & $\begin{array}{c}8.78 \\
(2.33)\end{array}$ & $\begin{array}{c}2.11 \\
(0.58)\end{array}$ & $\begin{array}{c}7.14 \\
(3.07)\end{array}$ & $\begin{array}{c}9.26 \\
(3.43)\end{array}$ \\
\hline & 3 dígitos & $\begin{array}{c}2.09 \\
(0.74)\end{array}$ & $\begin{array}{c}4.28 \\
(3.45)\end{array}$ & $\begin{array}{c}6.38 \\
(3.91)\end{array}$ & $\begin{array}{c}3.52 \\
(1.92)\end{array}$ & $\begin{array}{c}6.42 \\
(2.76)\end{array}$ & $\begin{array}{c}9.94 \\
(4.16)\end{array}$ \\
\hline \multirow[b]{2}{*}{$4 .^{\circ} \cdot 6 .^{\circ}$} & 2 dígitos & $\begin{array}{c}4.18 \\
(0.93)\end{array}$ & $\begin{array}{c}8.57 \\
(0.53)\end{array}$ & $\begin{array}{c}12.75 \\
(1.26)\end{array}$ & $\begin{array}{c}4.10 \\
(1.04)\end{array}$ & $\begin{array}{c}8.14 \\
(0.37)\end{array}$ & $\begin{array}{c}12.27 \\
(1.07)\end{array}$ \\
\hline & 3 dígitos & $\begin{array}{c}3.84 \\
(1.83)\end{array}$ & $\begin{array}{c}7.57 \\
(2.93)\end{array}$ & $\begin{array}{l}11.41 \\
(4.14)\end{array}$ & $\begin{array}{c}2.77 \\
(1.24)\end{array}$ & $\begin{array}{c}5.57 \\
(2.76)\end{array}$ & $\begin{array}{c}9.20 \\
(3.00)\end{array}$ \\
\hline
\end{tabular}

: Figura 1
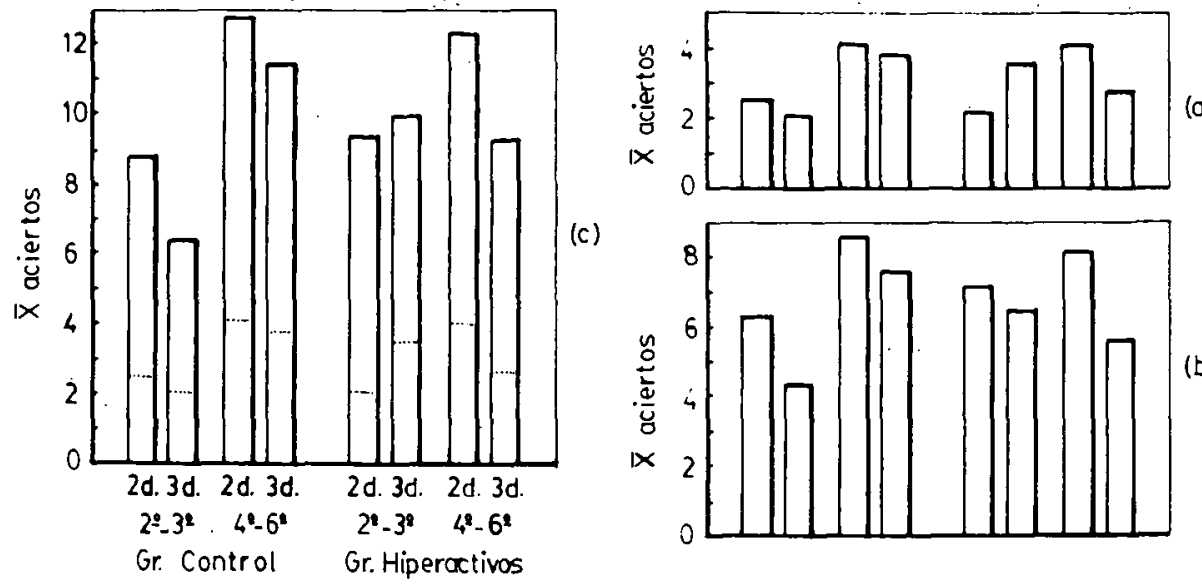

(c)

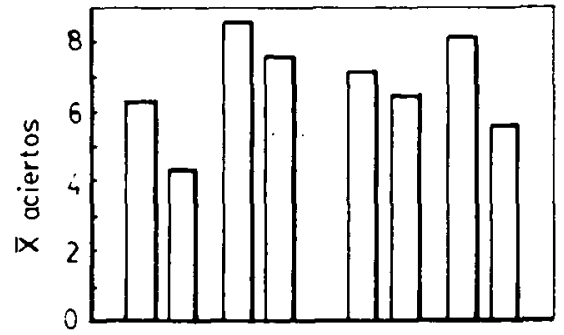

(b)

Diagrama de barras de la ejecución (media de aciertos) de los distintos grupos segün las puntuaciones en la tarea principal (a), tarea secundaria (b) y suma de ambas (c). $(2 d .=$ tarea de dos digitos, $3 d .=$ tarea de tres dígitos).

A nivel estadístico se comprueba la validez de estas afirmaciones al resultar $F$ significativas en el factor acursos en las tres puntuaciones $\left(P_{1}: F(1,48)=\right.$ 12.51, $\left.\mathrm{p}<.001 ; \mathrm{P}_{2}: \mathrm{F}(1,48)=4.67, \mathrm{p}<.05 ; \mathrm{P}_{\mathrm{i}}: \mathrm{F}(1,48)=11,27, \mathrm{p}<.01\right)$. La superioridad de la prueba de dos dígitos sólo es manifiesta estadísticamente en la puntuación obtenida de ella $\left(\mathrm{P}_{2}: \mathrm{F}(1,48)=5.66, \mathrm{p}<.05\right)$ pero no en su efecto principal e interferencia sobre la tarea principal (en $P_{1}$ el factor «dificultad de tareas no es significativo) aunque sí en su efecto secundario en interacción con «curso» $(\mathrm{F}(1,48)=4.09, \mathrm{p}<.05)$ por lo cual, tal y como se puede estudiar en la Figura 2.b, la mejora con la edad y curso en la ejecución de la tarea principal al realizar simultáneamente la tarea secundaria es mayor cuando ésta es más fácil que cuando es más difícil. De hecho, en la Figura 2.a, podemos apreciar que en los mayores se observa un descenso en la ejecución de la tarea principal cuando a la vez se realiza otra tarea más difícil. Sin embargo, en los cursos inferiores no se da este lógico descenso, sino una mala ejecución en ambas. 
FIGURA 2

(a)

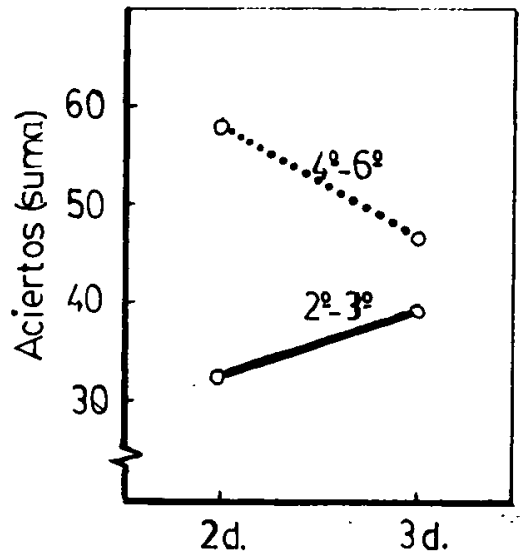

(b)

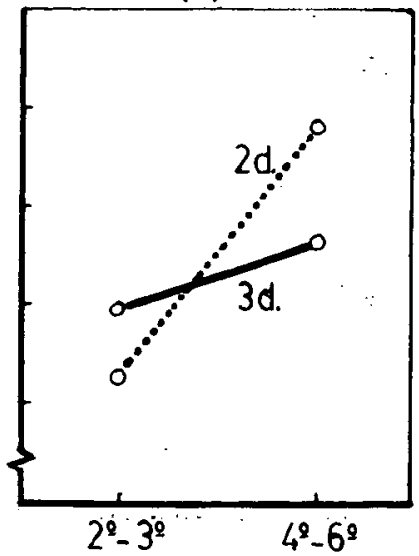

Representaciones gráficas de la interacción significativa "curso $\times$ difícultad de tarea secundaria al realizar la tarea principal $\left(P_{1}\right)$.

Este resultado puede analizarse mejor al considerar la vaiable «hiperactividad» en relación con las mencionadas anteriormente. En efecto, ninguno de los análisis de varianza muestran una diferencia significativa entre normales e hiperactivos, pero esta variable sí aparece como significativa en interacción con otras, en concreto con la variable "curso» y en las puntuaciones en la tarea secundaria $\left(\mathrm{P}_{2}: \mathrm{F}(1,48)=4.22, \mathrm{p}<.05\right)$ y en suma de ambas puntuaciones $\left(\mathrm{P}_{\mathrm{t}}\right.$ : $\mathrm{F}(1,48)=4.01, \mathrm{p}<.05)$. En ambos casos tienen los datos una misma interpretación: como muestra la Figura 3, los normales mejoran la ejecución con la edad y curso mucho más que los hiperactivos, los cuales apenas mejoran y obtienen paradójicamente en $2 .^{\circ}-3 .^{\circ}$ mejor puntuación que los normales, pero también (y de acuerdo con nuestras predicciones), mucho peor que éstos en $4 .^{\circ}-6^{\circ}$

Esta interacción «hiperactividad por curso» no se da solo al tener en cuenta la ejecución en la tarea principal sino a su vez en interacción con «dificultad de tarea secundarias $(F(1,48)=4.48, \mathrm{P}<.05)$. Tal y como se refleja en la Figura

FIGURA 3

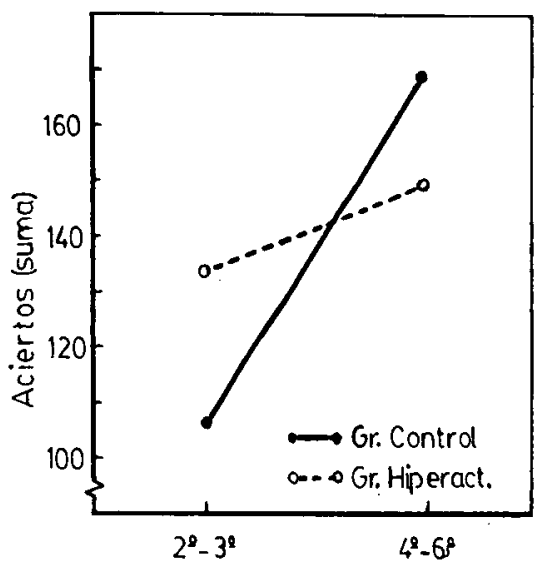

Representación gráfica de la interacción significativa "biperactividad $\times$ curso" en la tarea secundaria $\left(P_{2}\right)$. 
4, esta interacción significativa podrá despejar quizás la aparente rareza de la interacción acurso $\times$ dificultad de tarea secundarias en $P_{1}$ (Vid. Figura 2). En aquel caso, la ejecución mejora con la edad y curso al relizar concurrentemente una tarea fácil pero no otra tarea más difícil. Esto último puede entenderse si observamos la Figura 4.b: Todos los grupos de normales y el de hiperactivos

Figura 4

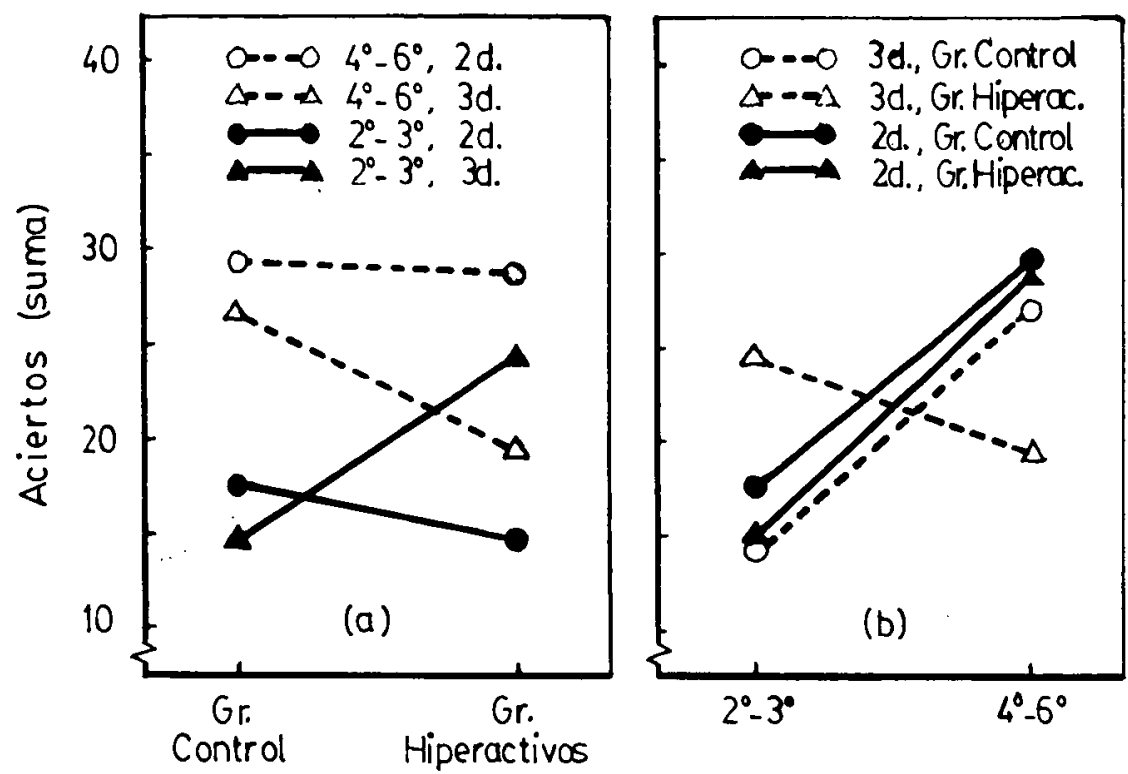

Representaciones gräficas de la interacción significativa "hiperactividad $\times$ curso $x$ dificultad en la tarea secundarian en puntuaciones sobre la tarea principal.

con tarea de dos dígitos incrementan de modo similar la ejecución con la edad y curso, pero no así el de hiperactivos de $2 .^{\circ}-3^{\circ}$ asignados a la tarea de tres dígitos. En este último grupo, sorprendentemente, los mayores tienen parecida ejecución que los menores y viceversa. Dicho de otro modo, y siguiendo la Figura 4.a: Al realizar la tarea secundaria fácil apenas hay diferencias en la ejecución de la tarea principal entre normales e hiperactivos de la misma edad y curso, pero al aplicar la tarea difícil, los hiperactivos mayores tienen peor rendimiento que los controles, pero estos tealizan peor la tarea principal que los hiperactivos, mientras ocurre justo lo contrario en $4.9-6.9$. Según nuestras predicciones iniciales, lo ocurrido en $4.0^{\circ}-6^{\circ}$ es lo correcto, o sea, los hiperactivos han tenido peor rendimiento que los controles, pero los resultados de $22^{\circ}-3 .^{\circ}$ con la tarea difícil no concuerdan con las mismas. La Figura 1.a pone esto de manifiesto: los hiperactivos de $2 .^{\circ} \cdot 3 .^{\circ}$ y con la prueba de tres dígitos obtienen mejor rendimiento que otros niños hiperactivos de $2 .^{\circ}-3 .^{\circ}$, pero con la prueba efácil» de dos dígitos. Entonces, ¿por qué ha interferido menos la tarea secundaria «difícil que la «fácil» y sólo en los hiperactivos más jóvenes?

\section{DISCUSION Y CONCLUSIONES}

Al exponer a los sujetos a un paradigma experimental de doble tarea hemos intentado poner a prueba algunos aspectos de los recientes modelos teóricos de la atención entendida como un mecanismo de limitación de la capacidad 
de procesamiento. Según la dificultad de la tarea, y por tanto según las limitaciones impuestas por los datos (estimulares o de memoria), o por los recursos de procesamiento, el sistema cognitivo se verá más o menos limitado para actuar, y ello se reflejará en la ejecución resultante. Nosotros hemos manipulado, precisamente, la dificultad de la tarea secundaria para observar hasta qué punto interfería en la ejecución de la tarea principal y por consiguiente hasta qué punto consume recursos de procesamiento, con el ánimo de averiguar si los niños hiperactivos difieren de los normales en este aspecto y por tanto si es posible caracterizar el déficit atencional de los hiperactivos en términos de una menor capacidad de asignación de recursos, o un sistema cognitivo con mayores limitaciones en su capacidad de actuación al enfrentarse a tareas auditivas y visuales.

De los resultados anteriormente descritos se pueden desprender los siguientes puntos de discusión:

1. Las diferencias en rendimiento entre normales e hiperactivos sólo son apreciables al considerar el nivel evolutivo (curso y edad), y ello ha quedado patente en la ausencia de significación estadística del factor principal shiperactividad» y en la interacción significativa de ésta con ucurso» (Vid. Figura 3) y del factor «curso» aisladamente en todas las puntuaciones. Los niños de cursos inferiores tienen peor ejecución que los de cursos superiores, y en la interacción con «hiperactividads, los normales mejoran con la edad y curso mientras que apenas lo hacen los hiperactivos (e incluso empeoran con la tarea fácil concurrente).

2. Cuando las tareas concurrentes no exceden los límites normales de la capacidad de procesamiento (tarea auditiva sfácils de dos dígitos) se obtienen unos patrones de resultados similares en normales y en hiperactivos, tanto en la tarea principal, en la secundaria concurrente como en la suma de ambas (Vid. Figura 1). Ahora bien, al incrementar la dificultad de la tarea secundaria y por tanto al necesitar mayor asignación de recursos de procesamiento, mayor esfuerzo y concentración. mayor focalización de la atención (según los distintos autores y modelos), las diferencias entre normales e hiperactivos se hacen más notorias.

3. El déficit atencional en cuanto a los límites de capacidad de procesamiento en los hiperactivos se hace patente al obtener éstos una ejecución discordante y de una mavor variabilidad que los normales ante tareas más difíciles y que exigen mayor asignación de recursos. En este caso, tal y como se puede apreciar en la Figura 1 , los hiperactivos mayores (de $4 .^{\circ}$ y $6 .^{\circ}$ ) tienen peor ejecución que los normales de igual curso y edad, con lo cual se cumple nuestra predicción inicial al respecto. Sin embargo, los hiperactivos menores (de $2 .^{\circ}$ y $3 .^{\circ}$ ) parecen tener incluso mayor capacidad y menos limitaciones en la asignación de recursos que los normales de igual curso y edad, es decir, en contra de nuestra predicción inicial, los hiperactivos tienen mejor ejecución que los normales a ese nivel de curso y edad. Además, con la edad, los hiperactivos mantienen igual ejecución intermedia. Este resultado anómalo podría justificarse en principio alegando problemas en el escaso tamaño de la muestra, pero no es un número pequeño si se compara con otras investigaciones y si se tienen en cuenta la gran meticulosidad en la selección psicométrica y clínica de los sujetos. Rechazada, pues, esta objeción metodológica, podemos esbozar los argumentos teóricos del siguiente punto de discusión.

4. La no mejora evolutiva de la ejecución en hiperactivos y la mejor ejecu. ción de éstos respecto a los normales de $2 .^{\circ}$ y $3 .^{\circ}$ podría explicarse si seguimos el modelo de Douglas y Peters (1979) antes expuesto. Recordemos que los tres síntomas del déficit atencional de los hiperactivos en su primera aparición (al- 


\section{8}

teración en atención sostenida, pobre control inhibitorio e impulsividad y «arousal pobremente moduladom), tras experiencias sucesivas de fracasos, llevaban a un incremento de síntomas y consecuencias negativas en un círculo vicioso que progresa con la edad. Si esto es así, es posible pensar que estos niños, en el inicio de la escolaridad no tienen límites de capacidad de procesamiento inferiores a los normales, cosa que se manifiesta, por otra parte, en otros hallazgos sobre no diferencias con normales en atención selectiva (Douglas y Peters, 1979; Prior et al., 1985; Sergeant y Scholten, 1985 a,b), incluso podría especularse que por su mayor impulsividad e índice de actividad tendrían mayor rapidez y menos limitaciones en asignación de recursos de procesamiento que los normales (en tareas motivadoras o reforzantes y de corta duración, tal como nuestra tarea experimental) pero que debido a la influencia de las experiencias de fracaso en otras actividades más sostenidas, con el tiempo hace que como consecuencia también se vaya reduciendo la capacidad en asignación de recursos tal y como se muestra en los hiperactivos de $4 .^{\circ}-6^{\circ}$ en nuestro experimento. No obstante, ante los posibles problemas metodológicos serían necesarias replicaciones ulteriores de estos resultados para reforzar la validez de estas conjeturas.

En definitiva, podríamos resumir este trabajo con las siguientes conclusiones:

1. El déficit atencional de los niños hiperactivos se puede caracterizar, a la luz de las últimas teorías atencionales de limitaciones en la capacidad de procesamiento, y según nuestros resultados, como una mayor labilidad de estos niños ante un aumento en la dificultad de tareas de procesamiento que se manifiesta de dos formas antagónicas según la edad y curso escolar: los menores tendrían una mejor disposición en la asignación de recursos de procesamiento que los normales, mientras que los mayores tendrían más limitaciones en dicha capacidad que los normales de igual edad y curso. El déficit atencional, expresado en términos de menor capacidad de procesamiento y por tanto en términos de una mayor dificultad en la asignación de recursos en el procesamiento «controlado» (Shiffrin y Schneider, 1977; Schneider y Shiffrin, 1977), consciente y que requiere esfuerzo, concentración y focalización atencional, sólo aparece en niños de 10 a 12 años, tal vez (siguiendo a Douglas y Peters, 1979), como resultado o consecuencia de fracasos repetidos en otros procesos atencionales y/o cognitivos.

2. Aunque las investigaciones básicas sobre los modelos atencionales de capacidad parten de formulaciones conceptuales y metodológicas más específicas y en mucho casos con un mayor aporte en modelos que derivan predicciones con una mayor precisión cuantitativa que lo reflejado en este trabajo (por ejemplo Botella, 1985; Kinchla, 1980; Navon y Gopher, 1979, 1980; Ponsoda, 1983: Wickens, 1980, 1984), al menos creemos que sí se puede afirmar que el énfasis mostrado por la nosología clínica actual (DSM III) en el «déficit atencional» de la hiperactividad no es desacertado, según se señala en las investigaciones experimentales al respecto, y que además en dicho déficit habría que incluir las anomalias de capacidad de procesamiento.

3. No obstante, sería necesario un mayor esfuerzo empírico en la aplicación de los recientes modelos básicos de la atención (Vid. Eysenck, 1982; Pasasuraman y Davies, 1984; Posner y Marin, 1985; para revisión) a los problemas clínicos en donde se han señalado déficits atencionales (hiperactividad, esquizofrenia, dificultades de aprendizaje, etc.). Con ello se lograría el objetivo señalado por Douglas y. Peters (1979) de una mayor precisión etiológica y una mejora en el diagnostico y tratamiento de la hiperactividad. 


\section{Notas}

1 Elaborado como test de recursos atencionales a partir de un estudio previo con adultos efec. tuado en este Departamento por M. Soriano, J. F. Martínez y M. V. Aguilar.

2 En concreto, pruebas de psicomotricidad, organización grafoperceptiva, personalidad, lectoescritura, etc., pero sus resultados conciernén a otra investigación distinta llevada a cabo por uno de los autores (López, 1968).

${ }^{3}$ Colegio Público «Narciso Yepess (Centro Piloto del I. C. E. de la Universidad de Murcia), a cuyos profesores y alumnos agradecemos la colaboración prestada para esta investigación.

\section{Referencias}

ACkerman, P. T.; ANHatal, J. M.; DYkman, R. A., y Holcomb, P. J. (1986). Effortful processing deficits in children with reading and/or attention disorders. Brain \& Cognition, S, 22-40.

A山PORT, D. A. (1980). Attention and performance. En G. Claxton (Ed.): Cognitive Psychology: new directions. Londres, Routledge \& Kegan Paul, 112-153.

American PSyChiatriac ASSOCIATION (APA) (1983). Diagnostic and Statistical Manual of Mental Disorders, III (DSM III). Washington, APA (Trad. española en Barcelona, Masson).

BoteLA J. (1985). Atención e interferencia entre estímulos y el estudio de la organización funcional de los procesos elementales. Revista de Psicología General y Aplicada, 40, 647-665.

Boums. H. (1978). Visual search and reading: Eye movements and functional visual field: A tutorial review. En J. Requin (Ed.): Attention \& Performance, VII. Hillsdale, N. J., L. Erlbaum.

BrondBent, D. E. (1958). Perception and communication. Londres, Pergamon. (Trad. española en Madrid, Debate, 1983).

- (1970). Stimulus set and response set: two kinds of selective attention. En D. Mostofsky (Ed.): Attention: Contemporary theories and Analysis. Nueva York, Appleton Century Croffts.

DeUTSCH, J. A. y DeUTSCH, D. (1963). Attentions: some theoretical considerations. Psychological Review, $70,80-90$.

DOUGLAS, J. A. y PETERS, K. G. (1979). Toward a clearer definition of the attentional deficit of hyperactive children. En G. A. Hale y M. Lewis (Eds.): Attention and cognitive development. Nueva York, Plenum, 173-248.

Duncan, J. (1980). The locus of interference in the perception of simultaneous stimuli. Psycbological Review, 87, 272-300 (Trad. española en Estudios de Psicología, 1981, 7, 58-90).

EYSENCK. M. W. (1982). Attention and arousal. Cognition and performance. Berlin, Spriger-Verlag. (Trad. española en Barcelona, Herder, 1985).

HASHER. L. y ZACKS, R. T. (1979). Automatic and effortful processes in memory. Joumal of Experimental Psychology: General, 108,356-388.

Hiscock. M.; Kinsbourne, M; Caplan, B. y Swanson, J. M. (1979). Auditory attention in hyperactive children: Effects of stimulant medication on dichotic listening performance. Journal of $\mathrm{Ab}$ normal Psychology, 88, 27-32.

JOHNSTON, W. A. y HEINZ, S. P. (1978). Flexibility and capacity demands of attention. Joumal of Experimental Psychology: General, 107, 420-435 (Trad. española en Estudios de Psicología, 1981, 7, 42-57).

Kahneman, D. (1973). Attention and effort. Englewood Cliffs, N. J., Prentice-Hall.

KINCHLA, R. A. (1980). The measurement of attention. En R. S. Nickerson (Ed.): Attention \& Performnace, VIII. Hillsdale, N. J., L. Erlbaum, 213-238.

LOPEZ, C. (1986). Estudio empinico de la disfusión cerebral minima (sindrome de biperactividad infantil): caracterización clínica y psicométrica). Tesis doctoral (no publicada). Univ. de Murcia.

NAVON, D. y GOPHER. D. (1979). On the economy of the human-processing system. Psychological Review, 86, 214-255.

- (1980). Task difficulty, resources, and dual-task performance. En R. S. Nickerson (Ed.): Attention \& Performance, VIII. Hillsdale, N. J.; L. Erlbaum, 297-315.

Norman, D. A. (1968). Toward a theory of memory and attention. Psychological Review, 75, \$22.536.

- (1976). Memory and attention (2.2 ed.). Nueva York, Wiley.

Norman, D. A. y BOBROw. D. G. (1975). On data-limited and resource-limite processes. Cognitive Psychology, 7, 44-64.

Parasuraman, R. y Davies, D. R. (Eds.). (1984). Varieties of attention. Nueva York, Academic.

PONSODA, V. (1983). Modelos de atención: modelo de canal único, de dos canales y modelo mixto. Anuario de Psicología, 29,:-91-106.

POSNER, M. I. y SNYDER, C. R. R. (1975). Attention and cognitive control. En R. Solso (Ed.). Information processing and cognition: The Loyola Symposium. Hillsdale, N. J., L. Erlbaum, 55-85. 
PRIOR, M. y GRIFIN, M. (1985). Hyperactivic: Diagnosis and management. Londres, Heinemann Medical Books.

Prior. M.; Sanson, A.; Freethy, C. y Gefren, G. (1985). Auditory attentional abilities in hyperactive children. Journal of Child Psychology \& Psychiatry, 26, 289-394.

Rosenthal, R. H. y ALLEN, T. W. (1978). An examination of attention, arousal, and learning dysfunctions of hyperkinetic children. Psychological Bulletin, 85, 689-715.

Ruiz-VARGAS. J. M. y Bơtella. J. (1981). Limitaciones de procesamiento y selectividad atencional. Estudios de Psicología, 7, 30-41.

SCHNEIDER. W. y SHIFFrIN, R. M. (1977). Controlled an automatic human information processing: I. Detection, search and attention. Psychological Review, 84, 1-66.

Sergennt. J. A. y SCholten. C. A. (1985a). On resource strategy limitatione in hyperactiviy: cognitive impulsivity reconsidered. Journal of Child Psychology \& Psychiatry, 26, 97-109.

- (1985b). On data limitations in hyperactivity. Joumal of Child Psychology \& Psychiatry, 26, 111-124.

Shiffrin, R. M. y SCHNEIDER, W. (1977). Controlled and automatic human information processin: II. Perceptual learning, automatic attending, and a general theory. Psychological Review, 84, $127-190$.

TAYIOR, E. (1980). Development of attention. En: M. Rutter (Ed.): Scientific foundations of developmental psychiatry. Londres, Heinemann Medical Books, 185-197.

Treisman, A. M. (1960). Contextual cues in selective listening. Quarterly Journal of Experimental Psychology, 12, 242-248.

- (1964). Verbal cues, language, and meaning in selective attention. American Jourmal of Psycho$\log y, 77,206-219$.

VEGA, M. DE. (1984). Introducción a la psicología cognitiva. Madrid, Alianza.

WELFORD, A. T. (1952). The psychological refractory period and the timing of highspeed performance: a review and a theory. British Joumal of Psychology, 43, 2-19.

- (1968). Fundamentals of skills. Londres, Methuen.

WICKENS. C. D. (1980). The structure of attentional resources, en R. S. Nickerson (Ed.): Attention \& Performance, VIII. Hillsdale, N. J., L. Erlbaum, 239-258.

- (1984). Processing resources in attention. En R. Parasuraman y D. R. Davies (Eds.): Varieties of attention. Nueva York, Academic, 63-101.

\section{Apéndice}

\section{CUESTIONARIO DE HIPERACTIVIDAD}

\section{Falta de atenciōn}

- A menudo no puede acabar las cosas que empieza

SI NO

- A menudo no parece escuchar

SI NO

- Se distrae con facilidad

SI NO

- Tiene dificultades para concentrarse en el trabajo escolar o en tareas que exigen atención sostenida

- Tiene dificultades para concentrarse en un juego

SI NO

SI NO

\section{Impulsividad}

- A menudo actúa antes de pensar

- Cambia con excesiva frecuencia de una actividad a otra

- Tiene dificultades para organizarse en el trabajo

- Necesita supervisión constantemente

- Le cuesta guardar turno en los juegos o en las situaciones grupales

\section{Sobreactividad}

- Corre de un lado para otro en exceso, o se sube a los muebles

- Le cuesta mucho quedarse quieto en un sitio o se mueve excesivamente

- Le cuesta estar sentado

- Se mueve mucho durante el sueño

- Está siempre «en marcha» o actúa «como si tuviese un moton 